Taiju Miyagami^, Yuki Uehara, Taku Harada, Takashi Watari, Taro Shimizu, Ayako Nakamura, Naoya Ogura, Seiko Kushiro, Katsutoshi Masuyama, Yoshinori Kanai, Kwang-Seok Yang and Toshio Naito

\title{
Delayed treatment of bacteremia during the COVID-19 pandemic
}

https://doi.org/10.1515/dx-2020-0114

Received August 15, 2020; accepted December 15, 2020; published online January 19, 2021

\begin{abstract}
Objectives: Coronavirus disease (COVID-19) blindness, that is, the excessive consideration of the disease in diagnosis, has reportedly led to delayed diagnosis of some diseases. We compared several clinical measures between patients admitted for bacteremia during the two months of the COVID-19 pandemic and those admitted during the same period in 2019. We hypothesized that the pandemic has led to delayed treatment of bacteremia.

Methods: This retrospective observational study compared several measures undertaken for patients who visited the emergency unit in two hospitals between March 1 and May 31, 2020, during the COVID-19 pandemic and whose blood cultures tested positive for bacteremia with those for corresponding patients treated during the same period in 2019. The primary measure was time from consultation to blood culture/antimicrobials.
\end{abstract}

*Corresponding author: Taiju Miyagami, MD, PhD, Department of General Medicine, Juntendo University Faculty of Medicine, 2-1-1 Hongo, Bunkyo-ku, Tokyo, Japan, Phone: +8133813 3111, Fax: +81 3 5802 1190, E-mail: tmiyaga@juntendo.ac.jp. https://orcid.org/00000002-4893-2224

Yuki Uehara, Department of General Medicine, Juntendo University Faculty of Medicine, Tokyo, Japan; and Department of Clinical Laboratory, St Luke's International Hospital, Tokyo, Japan Taku Harada, Division of General Medicine, Showa University Koto Toyosu Hospital, Tokyo, Japan; and Department of Diagnostic and Generalist Medicine, Dokkyo Medical University Hospital, Tochigi, Japan

Takashi Watari, Postgraduate Clinical Training Center, Shimane University Hospital, Izumo City, Shimane, Japan

Taro Shimizu, Department of Diagnostic and Generalist Medicine, Dokkyo Medical University Hospital, Tochigi, Japan

Ayako Nakamura and Naoya Ogura, Department of Clinical Laboratory, Juntendo Tokyo Koto Geriatric Medical Center, Tokyo, Japan

Seiko Kushiro, Katsutoshi Masuyama, Yoshinori Kanai, Kwang-Seok Yang and Toshio Naito, Department of General Medicine, Juntendo University Faculty of Medicine, Tokyo, Japan
Results: We included 29 eligible patients from 2020 and 26 from 2019. In 2020, the time from consultation to antimicrobial administration was significantly longer than in 2019 (mean [range], 222 [145-309] min vs. 139 [102-179] $\min , \mathrm{p}=0.002)$. The frequency of chest computed tomography (CT) was significantly higher in 2020 (96.6 vs. 73.1\%, $\mathrm{p}=0.021)$. Significant differences were not observed in the time to blood culture or chest CT preceding the blood culture between the two periods.

Conclusions: Our findings suggested that due to the COVID-19 epidemic/pandemic, focusing on the exclusion of its infection using CT scans leads to an overall delay in the diagnosis and treatment of bacteremia. Medical providers must be aware of COVID-19 blindness and evaluate patients objectively based on rational criteria and take appropriate action.

Keywords: bacteremia; COVID-19; COVID-19 blindness.

\section{Introduction}

Coronavirus disease (COVID-19) was declared a global epidemic in 2020. As of November 14, severe acute respiratory syndrome coronavirus 2 (SARS-CoV-2) has infected $52,686,373$ individuals worldwide and killed 1,292,733 people. The respective numbers for Japan are 113,649 and 1,879 , respectively [1]. In the past, the influenza pandemic of 1,918 killed an estimated 50 million people worldwide [2], and the bubonic plague pandemic of the 14th century may have killed up to 200 million people [3]. The scale of the damage caused by the pandemic is unprecedented for an infectious disease in modern times; however, as of November 2020, no end seems to be in sight for the contagion.

Some case reports have noted that the COVID-19 pandemic has disrupted the normal processes of clinical inference and diagnosis. For example, one patient with a myocardial infarction had a delayed diagnosis due to over concerns of potential SARS-CoV-2 infection [4]. Similar concerns led to delayed administration of antimicrobials in a case of bacteremia [5]. We speculate that the COVID-19 
epidemic has led to an overall delay in the diagnosis and treatment of bacteremia because of an overzealous attempt to always put COVID-19 at the top of the differential list and to exclude COVID-19 by computed tomography (CT) scan. If true, these shifts might have led to delays in phlebotomy for blood cultures and administration of antimicrobials in patients with bacteremia. To the best of our knowledge, no study has examined the treatment time course from consultation to blood culture and/or antimicrobial administration in patients with bacteremia and sepsis during the COVID-19 epidemic.

In Japan, the number of COVID-19 infections began to increase on February 20, 2020, which led to the suspension of public events by the Prime Minister on February $26[6,7]$. The aim of our study was to examine for differences in the performance of blood culture and treatment of bacteremia in 2019 as compared with that in similar cases treated during the 2 month COVID-19 period beginning March 1, 2020. Differences in the following a parameter at the emergency department was assessed: time from consultation to blood culture/antimicrobial administration.

\section{Materials and methods}

\section{Setting and patients}

This was a retrospective observational study of data retrieved from medical records. The data of patients who visited the emergency unit of Juntendo Tokyo Koto Geriatric Medical Center or Showa University Toyosu Hospital between March 1 and May 31, 2020, and whose blood cultures were positive for bacteremia were extracted. Their data were compared with those of patients with bacteremia who had positive blood cultures at the two centers during the same period in 2019 (March 1, 2019-May 31, 2019). The exclusion criteria were (1) unknown time of blood sampling and/or antimicrobial administration; (2) contaminated blood culture; (3) missing data; (4) blood culture and/or administration of antimicrobials after hospitalization, not at the emergency department; and (5) CT scan(s) already performed at a different center/clinic. The dataset obtained was password protected and managed by the three authors.

\section{Ethical consideration}

The Institutional Ethics Committee of Juntendo Tokyo Koto Geriatric Medical Center approved the study protocol (No. 106-9). We posted the consent form on the website and obtained consent from all the participants.

\section{Survey instrument}

Basic information included patient age and sex, bacterial species that caused the infection, underlying diseases (i.e., diabetes, chronic kidney disease, and dementia), and current drug use (i.e., immunosuppressants, prednisolone, and anticancer drugs). The parameters used in the Quick Sepsis Related Organ Failure Assessment (qSOFA), namely hypotension (systolic blood pressure $\leq 100 \mathrm{mmHg}$ ) on arrival, increased respiratory rate ( $\geq 22 \mathrm{bpm}$ ) on arrival, and disturbed consciousness on arrival, were also recorded [8]. The frequency of additional tests performed under the suspicion of COVID-19 was also compared, including urinary antigens (e.g., Legionella, Pneumococcus and Mycoplasma), blood biochemical indicators (e.g., brain natriuretic peptide and sialylated carbohydrate antigen KL-6, ferritin, procalcitonin, and blood gas). Some patients in the cohort died after hospitalization. The time from consultation to blood culture/antimicrobial administration was checked in electronic medical records. Two measures related to chest CT scans were also evaluated: frequency (i.e., the proportion of all cases who were examined by chest CT) and rate of precedence (i.e., the proportion of all cases in whom chest CT preceded phlebotomy for blood culture).

Blood cultures were performed using the same models of automated analysis system and resin-containing bottles (BD BACTEC FX and BD BACTEC Aerobic/23F + Anaerobic/22F, Nippon Becton Dickinson, Tokyo, Japan).

\section{Data collection}

Patients whose blood cultures were contaminated were excluded from the dataset prior to the analysis. Contamination was defined as the presence of at least one of the following bacteria: coagulase-negative staphylococci, Corynebacterium spp. (other than C. jeikieum), Bacillus spp., Propionibacterium acnes, viridans group streptococci, or Clostridiium perfringens, or a positive culture $>72 \mathrm{~h}$ after injection into the culture medium [9].

\section{Data analysis}

Continuous variables are presented as median values and interquartile ranges (IQRs), and categorical variables are presented as the number and proportion (\%) of corresponding cases. Categorical variables were compared using the $\chi^{2}$-test. Continuous variables were compared using the Student's t-test (normally distributed data) or Mann-Whitney U-test (non-normal distributions). The JMP PRO v. 13.0 software (SAS Institute, Cary, NC, USA) was used for all the calculations.

\section{Results}

The total number of blood culture drawn of 2020 at Juntendo was 106 and at Showa was 62, 2019 at Juntendo was 123 and at Showa was 89. Excluding cases of contamination, positive blood cultures were found in 18 of the 106 patients from the Juntendo center (17.0\%) and in 14 of 62 patients from the Showa hospital (22.5\%) during the 2020 study period. Positive blood culture cases were not significantly different from 2020 and 2019 ( $\mathrm{p}=0.21)$. Furthermore, there were no polymerase chain reaction (PCR)-positive cases of COVID-19. Of the cases, 29 positive cases met the inclusion criteria 
(Juntendo: 17/18, Showa: 11/15). During the corresponding 2019 period, positive blood cultures were recorded in 15 of 123 patients from the Juntendo center (12.2\%) and 15 of 89 patients from the Showa hospital (16.9\%). Of these patients, 26 with positive cultures met the inclusion criteria (Juntendo: 13/16, Showa: 13/15). Some patients had infections caused by multiple bacterial species; in all, 32 and 27 species were detected in the 2020 and 2019 cohorts, respectively (Figure 1).

The median age was 82 years (IQR: 71-89) in the 2020 group and 83 years (77-88) in the 2019 group. The 2020 and 2019 groups included 11 (37.9\%) and 12 men (46.2\%), respectively. In the 2020 group, eight patients had a qSOFA score of $\geq 2$ points $(27.6 \%)$, and in the 2019 group, nine patients had a qSOFA score of $\geq 2$ points (34.6\%). Two patients in the 2020 group died (6.9\%), and one died in the 2019 group (3.9\%). Comorbid pneumonia was found in one patient in $2020(3.5 \%)$ and in three patients in 2019 (12.0\%; Table 1).

In 2020, the time to administration of antimicrobials was significantly longer than in 2019 (mean [IQR], 222 [145-309] min vs. 139 [102-179] $\mathrm{min}, \mathrm{p}=0.002$ ), and the frequency of chest CT was significantly higher (96.6 vs. $73.1 \%, \mathrm{p}=0.021)$. However, the time to blood culture was comparable between the groups (2020: 70 [37-115] min vs. 2019: 68 [38-120] min, $p=0.91)$. Finally, chest CT preceded blood culture more often in 2020 than in 2019; however, the difference was not significant (58.6 vs. $34.6 \%, \mathrm{p}=0.11$ ). No significant differences were observed in the frequencies of any other clinical tests examined (Table 2).

Escherichia coli were the most common pathogen identified in both groups (2020: 40.6\% and 2019: 37.0\%). The most common site of infection in 2020 was the hepatobiliary system (31.0\%), while it was the urinary tract in 2019 (53.8\%) (Table 3).

\section{Discussion}

Our findings suggest that the COVID-19 pandemic has delayed the initiation of antimicrobial treatment for bacteremia and increased the frequency of chest CT examinations in hospitals. Significant differences were not observed in the time to blood culture or chest CT preceding the blood culture between the two periods.

A previous study recommended that patients with bacteremia with qSOFA scores $\geq 2$ should receive antimicrobial drugs within $1 \mathrm{~h}$ of hospital arrival [10]. However, no definitive window for "time to antimicrobials" has been established, as the literature on antimicrobial therapy for bacteremia mentions little beyond advocating early initiation [11]. Our study confirmed that in the patients with bacteremia, administration of antimicrobials was initiated later after arrival in 2020 than in 2019, although the clinical processes followed from diagnosis to treatment were not different. Studies have shown that patients admitted to the hospital for bacteremia are more likely to die within $48 \mathrm{~h}$ than those admitted for non-bacteremic conditions [11], and other study reported that that early intervention $(<3 \mathrm{~h})$ reduces the mortality rate in cases of bacteremia [12]. Hence, early action is crucial in cases of suspected bacteremia. Besides, the urgency of initiating treatment for bacteremia should be emphasized, regardless of COVID-19 status concerns.

We believe that the fact that the 2020 group underwent higher chest CT examinations despite the lower incidence of pneumonia is a result of the high sensitivity and specificity of mortality in detecting early-stage COVID-19 [13, 14]. We could hypothesize that the patients' attending physicians asked for chest CT to rule out COVID-19, which might have affected the time elapsed until antimicrobial administration.
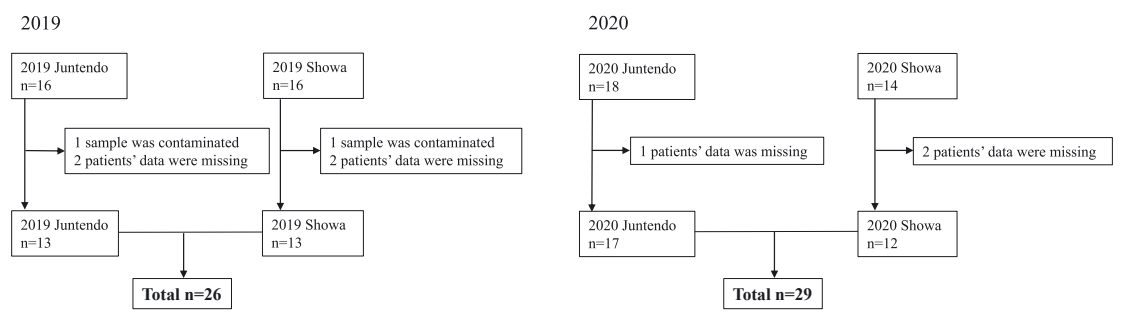

Figure 1: Eligibility of the study participants.

The patients with positive blood culture results during the study period in 2020 and corresponding period in 2019 were screened in Juntendo Tokyo Koto Geriatric Medical Center (Juntendo) and Showa University Toyosu Hospital (Showa). After excluding the patients with missing clinical data or cultures contaminated with microorganisms, eligible patients were selected. The details of the missing clinical data were as follows: (1) time of blood sampling and/or antimicrobial administration not recorded; (2) blood culture and/or antimicrobials administered after hospitalization; and (3) CT scans already performed at a different center/clinic. 
Table 1: Patients' characteristics.

\begin{tabular}{|c|c|c|c|}
\hline Parameters & $\begin{array}{r}2019 \\
(n=26)\end{array}$ & $\begin{array}{r}2020 \\
(n=29)\end{array}$ & p-Val \\
\hline Men, n (\%) & $12(46.2)$ & $11(37.9)$ & \\
\hline Age, years; mean (IQR) & $83(77-88)$ & $82(71-89)$ & \\
\hline \multicolumn{4}{|l|}{ Current medical history } \\
\hline DM, n (\%) & 9 (34.6) & 7 (24.1) & \\
\hline CKD, n (\%) & $2(7.7)$ & $0(0)$ & \\
\hline Dementia, n (\%) & $6(23.0)$ & $11(37.9)$ & \\
\hline On prednisolone, $\mathrm{n}(\%)$ & 0 & 0 & \\
\hline $\begin{array}{l}\text { On immunosuppressants, } \\
\mathrm{n}(\%)\end{array}$ & $2(7.7)$ & 0 & \\
\hline \multicolumn{4}{|l|}{ qSOFA } \\
\hline $\begin{array}{l}\text { Respiratory rate } \geq 22 \text { bpm, } \\
\mathrm{n}(\%)\end{array}$ & $10(38.5)$ & $12(41.4)$ & \\
\hline $\begin{array}{l}\text { Blood pres } \\
\text { sure } \leq 100 \mathrm{mmHg}, \mathrm{n}(\%)\end{array}$ & 7 (26.9) & $4(13.8)$ & \\
\hline $\begin{array}{l}\text { Altered consciousness, } \\
\mathrm{n}(\%)\end{array}$ & $7(26.9)$ & $8(27.6)$ & \\
\hline $\mathrm{qSOFA} \geq 2, \mathrm{n}(\%)$ & $9(34.6)$ & $8(27.6)$ & 0.7 \\
\hline \multicolumn{4}{|l|}{ Frequency of additional testing } \\
\hline $\begin{array}{l}\text { Influenza rapid antigen, } \\
\text { nasopharynx, } \mathrm{n}(\%)\end{array}$ & $21(80.1)$ & $14(48.3)$ & \\
\hline $\begin{array}{l}\text { Mycoplasma pneumoniae } \\
\text { rapid antigen, throat, } \mathrm{n}(\%)\end{array}$ & $6(23.1)$ & $2(6.9)$ & \\
\hline $\begin{array}{l}\text { Streptococcus pneumo } \\
\text { niae rapid antigen, urine, } \\
\mathrm{n}(\%)\end{array}$ & $10(38.5)$ & $6(20.7)$ & \\
\hline $\begin{array}{l}\text { Legionella rapid antigen, } \\
\text { urine, } n(\%)\end{array}$ & $8(36.4)$ & $4(16.0)$ & \\
\hline BNP, n (\%) & $2(7.7)$ & $10(34.5)$ & 0.02 \\
\hline KL-6, n (\%) & 0 & $1(3.5)$ & \\
\hline Sputum culture, n (\%) & $11(42.3)$ & $9(31.0)$ & \\
\hline Ferritin, n (\%) & $1(3.5)$ & $1(3.5)$ & \\
\hline Procalcitonin, n (\%) & $3(12.0)$ & $7(24.1)$ & \\
\hline Deaths, n (\%) & $1(3.9)$ & $2(6.9)$ & \\
\hline $\begin{array}{l}\text { Comorbid pneumonia, } \\
\mathrm{n}(\%)\end{array}$ & $3(12.0)$ & $1(3.5)$ & \\
\hline
\end{tabular}

Values are presented as median (interquartile range) or number (\%). $I Q R$, interquartile range; DM, diabetes mellitus; CKD, chronic kidney disease; qSOFA, quick sequential organ failure assessment; BNP, blood natriuretic peptide.

Recent studies conducted after the COVID-19 outbreak has reported high out-of-hospital mortality due to acute coronary syndrome $[15,16]$ and exacerbation of giant cell arteritis [16]. One reason might be that patients postponed their hospital visits because of fear of contracting COVID-19 [17]. Moreover, the higher burden on medical practitioners due to the pandemic can lead to more diagnostic errors. Prepandemic studies have reported that unusual situations can disrupt diagnostic protocols because of the associated workload and time pressures [18]. In fact, one study among nurse practitioners found high workload to be associated with a higher incidence of adverse events $[19,20]$. Moreover,
Table 2: Time from consultation and frequency rates of blood culture, antibiotic administration, and chest CT.

\begin{tabular}{lrrr}
\hline & $\begin{array}{r}\mathbf{2 0 1 9} \\
(\mathbf{n = 2 6})\end{array}$ & $\begin{array}{r}\mathbf{2 0 2 0} \\
(\mathbf{n = 2 9 )}\end{array}$ & p-Value \\
\hline $\begin{array}{l}\text { Time to blood culture, } \\
\text { min (IQR) }\end{array}$ & $68(38-120)$ & $70(37-115)$ & 0.91 \\
$\begin{array}{l}\text { Time to antibiotics, } \\
\text { min (IQR) }\end{array}$ & $139(102-179)$ & 222 & 0.002 \\
$\begin{array}{l}\text { Chest CT preceded } \\
\text { blood culture, } \mathrm{n}(\%)\end{array}$ & $9(34.6)$ & $17(58.6)$ & 0.11 \\
$\begin{array}{l}\text { Chest CT performed, } n(\%) \\
\text { Blood gas analysis }\end{array}$ & $19(73.1)$ & $28(96.6)$ & 0.021 \\
performed, $n$ (\%) & $19(76.0)$ & $19(65.5)$ & 0.55 \\
\hline
\end{tabular}

Values are presented as median (interquartile range) or number (\%). $I Q R$, interquartile range. The analysis of the time to blood culture was performed in 22 cases for 2019 and in 25 cases for 2020 , because the data was not available.

Table 3: The "top 3" bacterial species and infection foci.

\begin{tabular}{|c|c|c|c|}
\hline Bacterial species & $\begin{array}{r}2019 \\
(n=27)\end{array}$ & & $\begin{array}{r}2020 \\
(n=32)\end{array}$ \\
\hline $\begin{array}{l}\text { Escherichia coli, } \\
\text { n (\%) }\end{array}$ & $10(37.0)$ & Escherichia coli, n (\%) & $13(40.6)$ \\
\hline $\begin{array}{l}\text { Klebsiella } \\
\text { pneumoniae, n (\%) }\end{array}$ & $4(14.8)$ & $\begin{array}{l}\text { Klebsiella } \\
\text { pneumoniae, n (\%) }\end{array}$ & $5(15.6)$ \\
\hline $\begin{array}{l}\text { Staphylococcus } \\
\text { aureus, n (\%) }\end{array}$ & $3(11.1)$ & $\begin{array}{l}\text { Staphylococcus } \\
\text { aureus, } \mathrm{n}(\%)\end{array}$ & $3(9.4)$ \\
\hline Infection type & $\begin{array}{r}2019 \\
(n=26)\end{array}$ & & $\begin{array}{r}2020 \\
(n=29)\end{array}$ \\
\hline $\begin{array}{l}\text { Urinary tract } \\
\text { infection, n (\%) }\end{array}$ & $14(53.8)$ & $\begin{array}{l}\text { Hepatobiliary } \\
\text { infection, n (\%) }\end{array}$ & $9(31.0)$ \\
\hline $\begin{array}{l}\text { Hepatobiliary } \\
\text { infection, n (\%) }\end{array}$ & $5(19.2)$ & $\begin{array}{l}\text { Urinary tract } \\
\text { infection, } \mathrm{n}(\%)\end{array}$ & $8(27.6)$ \\
\hline Pneumonia, n (\%) & $3(9.4)$ & $\begin{array}{l}\text { Skin/soft tissue } \\
\text { infection, n (\%) }\end{array}$ & $3(10.3)$ \\
\hline
\end{tabular}

Values are presented as number (\%).

difficult-to-diagnose cases require a higher number of diagnostic tests [21]. Health-care providers should be aware of the inherent risks in such circumstances.

\section{Limitations}

The present study has several limitations. First, the best clinical practices might change depending on the spread of COVID-19. Second, our analysis might have been affected by the inadequate sample size, which might have led to a lack of statistical insignificance despite numerical 
differences in some measures between the cohorts. Third, institutional practices can vary greatly among hospitals depending on the number of CT systems available and testing fatigue among the staff. Forth, there were substantial differences in the sites of infection between 2019 and 2020. Hepatobiliary infections were more than twice as common in 2020 than in 2019, and the opposite was true for UTI's. This could have affected the results.

\section{Conclusions}

This study shows that patients with bacteremia who had positive blood cultures during the COVID-19 pandemic likely experienced delays in the time from hospital arrival to antimicrobial administration and subjected to chest CT examinations regardless of the pretest probability as compared with similar patients during the same period in 2019. During the COVID-19 pandemic, and in emergency situations, better awareness of the risks of disruptions to diagnostic and therapeutic protocols among medical providers is critical.

Acknowledgments: We thank Editage (www.editage.com) for the English language editing.

Research funding: A Japanese rock band named GLAY donated funds to this research. However, this research has no conflict of interest.

Author contributions: All authors accepted responsibility for the entire content of this manuscript and approved its submission.

Competing interests: Authors state no conflict of interest. Informed consent: Informed consent was obtained from all individuals included in this study.

Ethical approval: The Institutional Ethics Committee of Juntendo Tokyo Koto Geriatric Medical Center approved the study protocol (No. 106-9). We posted the consent form on the website and obtained consent from all the participants.

\section{References}

1. Ministry of Health LaWcJ. Tokyo. Coronavirus disease (COVID-19) situation within and outside the country November 142020 [Online]. Available from: https://www.mhlw.go.jp/stf/ seisakunitsuite/bunya/0000164708_00001.html [Updated 4 Jan 2021, Accessed 4 Jan 2021].

2. Centers for Disease Control and Prevention. Atlanta. Centers for Disease Control and Prevention, National Center for Immunization and Respiratory Diseases (NCIRD). History of 1918 flu pandemic [Online]. Available from: https://www.cdc.gov/flu/pandemicresources/1918-commemoration/1918-pandemic-history.html [Updated 21 Mar 2018, Accessed 4 Jan 2021].

3. Perry RD, Fetherston JD. Yersinia pestis-etiologic agent of plague. Clin Microbiol Rev 1997;10:35-66.

4. Yousefzai R, Bhimaraj A. Misdiagnosis in the COVID era: when zebras are everywhere, don't forget the horses. JACC Case Rep 2020;2:1614-9.

5. Brown L. COVID blindness. Diagnosis 2020;7:83-4.

6. Ministry of Health LaWcJ. Tokyo. Request for cooperation with labor and management organizations [Online]. Available from: https://www.mhlw.go.jp/stf/seisakunitsuite/newpage_ 00007.html [Updated 26 Feb 2020, Accessed 4 Jan 2021].

7. Welfare IMoHLa. Tokyo. A message to the public about the event Feb, 26, 2020 [Online]. Available from: https://www.mhlw.go.jp/ stf/seisakunitsuite/newpage_00002.html [Updated 20 Mar 2020, Accessed 4 Jan 2021].

8. Singer M, Deutschman CS, Seymour CW, Shankar-Hari M, Annane D, Bauer M, et al. The third international consensus definitions for sepsis and septic shock (sepsis-3). Jama 2016; 315:801-10.

9. Hall KK, Lyman JA. Updated review of blood culture contamination. Clin Microbiol Rev 2006;19:788-802.

10. Cecconi M, Evans L, Levy M, Rhodes A. Sepsis and septic shock. Lancet 2018;392:75-87.

11. Søgaard M, Nørgaard M, Pedersen L, Sørensen HT, Schønheyder HC. Blood culture status and mortality among patients with suspected community-acquired bacteremia: a population-based cohort study. BMC Infect Dis 2011; 11:139.

12. Papadimitriou-Olivgeris M, Perdikis K, Cois M, Roth L, Mykoniati $\mathrm{S}$, Nusbaumer $\mathrm{C}$, et al. Predictors for delayed antibiotic administration among bacteraemic patients in the Emergency Department: differences between medical and surgical interns. Eur J Clin Invest 2020;50:e13324.

13. Fang $\mathrm{Y}$, Zhang H, Xie J, Lin M, Ying L, Pang P, et al. Sensitivity of chest CT for COVID-19: comparison to RT-PCR. Radiol 2020:200432. https://doi.org/10.1148/radiol. 2020200432.

14. Kanne JP. Chest CT findings in 2019 novel coronavirus (2019-nCoV) infections from Wuhan, China: key points for the radiologist. Radiol 2020;295:16-7.

15. Baldi E, Sechi GM, Mare C, Canevari F, Brancaglione A, Primi R, et al. Out-of-hospital cardiac arrest during the Covid-19 outbreak in Italy. N Engl J Med 2020;383:496.

16. De Filippo O, D'Ascenzo F, Angelini F, Bocchino PP, Conrotto F, Saglietto A, et al. Reduced rate of hospital admissions for ACS during Covid-19 outbreak in Northern Italy. N Engl J Med 2020; 383:88-9.

17. Monti S, Delvino P, Bellis E, Milanesi A, Brandolino F, Montecucco C. Impact of delayed diagnoses at the time of COVID-19: increased rate of preventable bilateral blindness in giant cell arteritis. Ann Rheum Dis 2020;79:1658-9.

18. Singh H, Graber ML. Improving diagnosis in health care-the next imperative for patient safety. N Engl J Med 2015;373: 2493-5. 
19. Oliveira AC, Garcia PC, Nogueira LS. Nursing workload and occurrence of adverse events in intensive care: a systematic review. Rev Esc Enferm USP 2016;50:683-94.

20. Aiken LH, Sloane DM, Bruyneel L, Van den Heede K, Griffiths $P$, Busse R, et al. Nurse staffing and education and hospital mortality in nine European countries: a retrospective observational study. Lancet 2014;383:1824-30.

21. Meyer AN, Payne VL, Meeks DW, Rao R, Singh H. Physicians' diagnostic accuracy, confidence, and resource requests: a vignette study. JAMA Int Med 2013;173:1952-8. 